\title{
Levels of $17 \beta$-hydroxysteroid dehydrogenase type 10 in CSF are not a valuable biomarker for multiple sclerosis
}

\author{
Zdenka Kristofikova*,1, Jan Ricny' ${ }^{1}$, Daniel Kaping ${ }^{1}$, Jan Klaschka² , Jolana Kotoucova ${ }^{1}$ \& \\ Ales Bartos ${ }^{1,3}$ \\ ${ }^{1}$ National Institute of Mental Health, 25067 Klecany, Czech Republic \\ ${ }^{2}$ Institute of Computer Science, Academy of Sciences, 18207 Prague, Czech Republic \\ ${ }^{3}$ Department of Neurology, Third Faculty of Medicine, University Hospital Kralovske Vinohrady, Charles University, 10034 Prague, \\ Czech Republic \\ *Author for correspondence: Tel.: +420 283088 246; zdenka.kristofikova@nudz.cz
}

\begin{abstract}
Aim: We aimed to characterize the role of mitochondrial $17 \beta$-hydroxysteroid dehydrogenase type 10 (17 $\beta$ HSD10) overexpression in multiple sclerosis (MS) and to evaluate its use as a biomarker. Materials \& methods: We estimated levels of 17 $\beta$-HSD10, amyloid $\beta$ 1-42, cyclophilin D, 17 $\beta$-HSD10-cyclophilin D complexes or $17 \beta$-HSD10-parkin complexes in cerebrospinal fluid (CSF) samples. Results: The increase in $17 \beta-H S D 10$ levels or in $17 \beta$-HSD10-parkin complexes and links to leukocytes were found only in relapsing-remitting MS. The sensitivity of the biomarker was $64 \%$, the specificity equaled $60-63 \%$ compared with controls. Conclusion: Increased CSF levels of $17 \beta-$ HSD10 in later stages of MS could be interpreted via its upregulation in demyelinated neuronal axons. CSF levels of $17 \beta$-HSD10 are not the valuable biomarker for the early diagnosis or for the progression of MS.
\end{abstract}

First draft submitted: 19 February 2018; Accepted for publication: 24 July 2018; Published online: 6 December 2018

Keywords: $17 \beta$-hydroxysteroid dehydrogenase type 10 - Alzheimer's disease • CSF biomarkers • cyclophilin D - diagnosis • mitochondrial dysfunction • multiple sclerosis $\bullet$ neuroinflammation $\bullet$ parkin $\bullet$ protein interactions

Multiple sclerosis (MS) is the most common neurological disorder affecting the CNS. Although the causes of MS are unknown, it is believed to occur as a result of genetic and environmental factors and damage of CNS can be caused, at least at part, by attacks of the body's own immune system. Current studies indicate that both the adaptive immune system and the innate immune system are involved. The pathology of MS is characterized by multifocal lesions also called plaques, by neuroinflammation, and by the destruction of myelin sheaths of neurons. However, links among neuroinflammatory and neurodegenerative components of the disease are still a matter of debate [1,2].

It is believed that secondary mitochondrial dysfunction observed in MS, and probably mediated by primary demyelination and neuroinflammation, markedly influences the progression of the disease. Mitochondrial dysfunction appears to be associated with T-cell and B-cell inflammation and microglia or macrophage activation, as well as with the release of proinflammatory cytokines. These cytokines (particularly TNF- $\alpha$ ) impede mitochondrial oxidative phosphorylation and associated ATP production (among others via reduction of mitochondrial complex I activity) and instigate mitochondrial reactive oxygen species production. This culminates in mitochondrial membrane permeabilization, altered mitochondrial dynamics and can result in cell death [3]. Variation in mitochondrial deoxyribonucleic acid and in nuclear-encoded mitochondrial genes may also contribute to MS susceptibility via reduction in ATP production. Experimental data obtained from patients with MS or in corresponding animal models indicate that mitochondrial density is increased in neuronal axons and astrocytes in all MS lesions at all stages, probably via compensatory mechanisms for increase in cellular energy demand [4-8].

The nucleus-encoded mitochondrial matrix protein 17 $\beta$-hydroxysteroid dehydrogenase type 10 (17 $\beta$-HSD10, EC 1.1.1.178/35/239/159/150) operates via multiple enzymatic as well as nonenzymatic functions. Its deficiency, overexpression or loss of function is associated with many and various pathologies [9]. Experiments on transgenic animals overexpressing 17ß-HSD10 and displaying higher baseline ATP levels have demonstrated a protective

Future $\because$ Medicine 
phenotype in a pharmacological model of Parkinson's disease (PD) or of oxidative/metabolic stress [10,11]. Direct interactions of cytosolic $17 \beta-H S D 10$ and cytosolic protein parkin (the component of a multiprotein E3 ubiquitin ligase complex, mutations in parkin are the most common causes of familial PD) appear to be involved in the regulation of mitochondrial levels of $17 \beta-\mathrm{HSD} 10$. For example, parkin overproduction increases levels of $17 \beta$ HSD10, PARK2 gene downregulation or deficiency causes $17 \beta-$ HSD10 depletion [12]. Cytosolic 17ß-HSD10 is imported into the mitochondrial matrix via the translocase of the outer mitochondrial membrane and the translocase of the inner mitochondrial membrane. Its regulation by parkin probably occurs through phosphatase and tensin homolog-induced putative kinase 1. It is suggested that the phosphatase and tensin homolog-induced putative kinase 1-mediated parkin pathway participates in clearance of dysfunctional mitochondria and that 17ß-HSD10 levels in mitochondria could be one of the mechanisms by which parkin preserves mitochondrial quality $[12,13]$.

In addition to parkin, 17 $\beta$-HSD10 is known as a binding partner of intracellular amyloid $\beta$ (A $\beta$ ) peptides, accumulated in mitochondria in Alzheimer's disease $(\mathrm{AD})$, which can lead to $\mathrm{A} \beta$-mediated mitochondrial dysfunction [9,11]. Under normal conditions, $17 \beta-$ HSD10 localized in the mitochondrial matrix binds cyclophilin $\mathrm{D}(\mathrm{cypD})$ and by preventing its translocation to the inner mitochondrial membrane can regulate the opening of the mitochondrial permeability transition pore (MPTP) mediated by cypD [14,15]. Under conditions of increased accumulation of mitochondrial $A \beta$, interactions of $A \beta$ and $17 \beta-H S D 10$ or those of $A \beta$ and cypD eliminate the regulation of cypD by $17 \beta-H S D 10$. Therefore, the translocation of cypD from the matrix and its interactions with MPTP are enhanced, which can lead to apoptosis/necrosis [14,15]. Moreover, $17 \beta$-HSD10 potentiates A $\beta$-induced cell stress in A $\beta$-rich environment [11]. Therefore, high 17 $\beta$-HSD10 overexpression in the brain of AD people does not seem to be protective, in contrast to PD or oxidative/metabolic stress, but can lead to mitochondrial dysfunction $[9,11,16]$. In addition to the above-mentioned unfavorable interactions of $17 \beta-\mathrm{HSD} 10$ and A $\beta$, high overexpression of $17 \beta-\mathrm{HSD} 10$ itself plays a role in AD pathogenesis. Namely, $17 \beta-\mathrm{HSD} 10$ protein is essential for the structural and functional integrity of mitochondria [17] but highly overexpressed 17ß-HSD10 may lead to mitochondrial dysfunction via mitochondrial matrix condensation and a partial loss of cristae structure [18].

Our previous studies were focused on evaluation of CSF levels of $17 \beta-H S D 10$, either total or bound to $A \beta$, as biomarkers of $\mathrm{AD}$ [19-21]. We have found that the total levels are the relatively high sensitive, but unfortunately not fully specific, biomarker for the early diagnosis of $\mathrm{AD}$ (significant increases in people with mild cognitive impairment due to $\mathrm{AD}$ and with $\mathrm{AD}$, the sensitivity to $\mathrm{AD} 80 \%$, the specificity $73 \%$ compared with nondemented controls and $53-59 \%$ compared with other types of dementia [21]). On the contrary, levels of $17 \beta-H S D 10-A \beta$ complexes were markedly dependent on neuroinflammation and thus were not the valuable biomarker for AD [20]. In this study, we decided to evaluate total levels of $17 \beta-\mathrm{HSD} 10$ in CSF as a biomarker for a neurodegenerative component of MS from the following reasons. First, the pathology of MS is characterized by a neurodegenerative component of disease $[1,2]$ and classical CSF biomarkers for $\mathrm{AD}$ (i.e., peptides/proteins associated with axonal and neuronal degeneration as A $\beta 1-42$, $\tau$ and phospho- $\tau$ ) are changed in MS and are evaluated as putative biomarkers for MS progression [22-24]. Second, enhanced total levels of 17ß-HSD10 in CSF, as a biomarker of protein overexpression in the brain, can reflect mitochondrial dysfunction and are the sensitive biomarker for the early diagnosis of AD [21]. Mitochondrial dysfunction influences the progression of MS [4-8]. And finally, we have observed increased CSF levels of total $17 \beta-H S D 10$ also in MS patients compared with age-matched controls [19]. However, we did not distinguish between MS in early and later stages, did not compare changes in 17ß-HSD10 with those in the most toxic fragment $A \beta 1-42$ and did not evaluate interactions of $17 \beta-H S D 10$ and of cytosolic protein parkin or of mitochondrial matrix protein cypD in order to characterize the role of $17 \beta-\mathrm{HSD} 10$ overexpression in MS in more details.

Aims of the study are as follows: to estimate CSF levels of $17 \beta-H S D 10$ in MS patients in early and later stages separately, to compare levels of $17 \beta$-HSD10 with those of $A \beta 1-42$, to estimate levels of $17 \beta$-HSD10-parkin

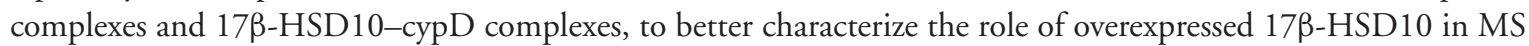
and finally to evaluate CSF levels of $17 \beta-\mathrm{HSD} 10$ as a biomarker for a neurodegenerative component of MS.

\section{Materials \& methods \\ Participants}

We collected CSF samples from a total of 120 individuals (62 patients with MS and 58 age- and gendermatched controls). The MS group was divided into the MS1 group $(n=26)$ and the MS2 group ( $n=36)$. MS1 patients were defined as those who had experienced their first clinical episode of neurological disturbance that was suggestive of MS (patients after the first attack). MS2 patients were people with relapsing-remitting MS (patients 
Table 1. Demographic characteristics of all patients providing CSF samples.

\begin{tabular}{llllllll|} 
Groups & $n(M / F)$ & Age (years) & Attacks, $n$ & EDSS & $\begin{array}{l}\text { Duration until LP } \\
\text { (months) }\end{array}$ & $\begin{array}{l}\text { Presence of } \\
\text { oligoclonal bands }\end{array}$ & $\begin{array}{l}\text { Oligoclonal bands, } n \\
\text { na }\end{array}$ \\
\hline SCs & $42(15 / 27)$ & $32.6 \pm 5.8$ & na & na & na & na & na \\
\hline INDCs & $16(6 / 10)$ & $36.9 \pm 6.9$ & na & na & na & na & na \\
\hline MS1 & $26(8 / 18)$ & $32.7 \pm 9.2$ & 1 & $1.7 \pm 0.6$ & $4.3 \pm 9.0$ & $96 \%$ & $10 \pm 5$ \\
\hline MS2 & $36(12 / 24)$ & $35.3 \pm 7.8$ & $5 \pm 4$ & $3.0 \pm 1.2$ & $47.9 \pm 45.2$ & $97 \%$ & $14 \pm 6$ \\
\hline
\end{tabular}

Data are presented as mean \pm standard deviation. Age was not significantly different across the groups (analysis of variance: $F[3,116]=1.94, p=0.128$ ).

CSF: Complexes in cerebrospinal fluid; EDSS: Expanded disability status score at the time of lumbal puncture; F: Female; INDC: Inflammatory neurological disease control; LP: Lumbar puncture; M: Male; MS: Multiple sclerosis; MS1: MS patients after the first attack; MS2: MS patients after at least two attacks; na: Not available; SC: Symptomatic control.

Table 2. More detailed clinical characteristic of patients from multiple sclerosis patients after at least two attacks group.

\begin{tabular}{|lllll|}
\hline Subgroups & $\mathrm{n}(\mathrm{M} / \mathrm{F})$ & Attacks, $\mathrm{n}^{\dagger}$ & EDSS & Treatment \\
$\begin{array}{l}\text { Relapsing-remitting form } \\
\text { (remission phase) }\end{array}$ & $16(6 / 10)$ & $2.2 \pm 0.8$ & $2.6 \pm 0.9$ & $10 \times$ none, 2x IM, 2x IS, 2x IM+IS \\
\hline $\begin{array}{l}\text { Relapsing-remitting form } \\
\text { (attack phase) }\end{array}$ & $20(6 / 14)$ & $3.3 \pm 2.9$ & $3.1 \pm 1.3$ & $\begin{array}{l}11 \times \text { none, } 2 \times I M, 5 \times I S, 1 \times I M+I S, ~ \\
1 \times \text { Tysabri }\end{array}$ \\
\hline ANOVA
\end{tabular}

Data are presented as mean \pm standard deviation.

$\dagger$ The number of attacks until lumbar puncture

ANOVA: Analysis of variance; EDSS: Expanded disability status score at the time of lumbal puncture; F: Female; IM: Immunomodulation drug; IS: Immunosuppressive drug; M: Male; MS: Multiple sclerosis.

having experienced at least two attacks). Patients in both groups met established criteria for MS at the time of lumbar puncture [25]. Controls were recruited from patients with heterogeneous neurological symptoms who had undergone a lumbar puncture as part of a routine diagnostic process. Controls were divided into symptomatic controls (SCs, 42 patients without objective clinical findings to define a specific neurological disease at the time of sampling, e.g., paraesthesias, vertigo, headache, etc.) and inflammatory neurological disease controls (inflammatory neurological disease controls [INDC], 16 patients with, e.g., neuroborreliosis, myelitis and aseptic meningitis, etc.), in accordance with guidelines [26]. Demographic characteristics (sex and age) of all patients are presented in Table 1. Table 2 shows clinical characteristics of MS2 patients in more details. All subjects gave written informed consent regarding study participation, in accordance with the Declaration of Helsinky. The Ethics Committee of the Third Faculty of Medicine, Charles University in Prague approved the study. Lumbar CSF samples were collected, examined, centrifuged, aliquoted in $1 \mathrm{ml}$ polypropylene tubes and stored until analysis (on average within $1.5 \mathrm{~h}$ from sampling) at $-80^{\circ} \mathrm{C}$ in accordance with established guidelines $[27,28]$. The specimens were thawed just prior to measurements.

\section{Methods}

\section{Competitive ELISA for estimation of CSF levels of $17 \beta-H S D 10$}

Measurements were performed in accordance with our previous study [21]. A fragment of human 17 $\beta$-HSD10 recombinant protein (amino acids 31-128, Abnova) was dissolved in carbonate buffer (75 $\mathrm{mM} \mathrm{NaHCO}_{3}, 25$ $\mathrm{mM} \mathrm{Na}_{2} \mathrm{CO}_{3}, \mathrm{pH}$ 9.5). The coating solution was applied to 96-well polystyrene plates (Nunc Immuno Plate Maxisorp, $42 \mathrm{ng}$ of protein per well) and incubated for $4 \mathrm{~h}$ at room temperature. Plates were subsequently washed three-times with phosphate buffer (2.8 $\mathrm{mM} \mathrm{Na}_{2} \mathrm{HPO}_{4}, 7.2 \mathrm{mM} \mathrm{NaH}_{2} \mathrm{PO}_{4}, 100 \mathrm{mM} \mathrm{NaCl}, \mathrm{pH}$ 7.2). Non-specific binding was suppressed by incubation with blocking solution $(10 \mathrm{mg} / \mathrm{ml}$ of bovine serum albumin in carbonate buffer) for $1 \mathrm{~h}$ at room temperature, followed by overnight incubation at $10^{\circ} \mathrm{C}$. Prior to the assay, the plates were washed three-times with washing solution (phosphate buffer containing $0.05 \%$ Tween 20). A standard curve was prepared using 0.9-222.2 ng of partial recombinant protein dissolved in 10\% dimethyl sulfoxide. About $100 \mu \mathrm{l}$ of standards and samples ( $40 \mu \mathrm{l}$ of CSF; volumes were adjusted to $100 \mu \mathrm{l}$ with $10 \%$ dimethyl sulfoxide) were incubated in duplicate with primary antibody (mouse polyclonal antibody against partial recombinant protein 29128, Abnova, dilution 1:6000) for $4 \mathrm{~h}$ at room temperature. Plates were washed four-times with washing solution, and secondary antibody (polyclonal rabbit antimouse immunoglobulin/HRP, DakoCytomation, dilution 1:6000) was added. After a 1-h incubation at room temperature, the plates were washed four-times with washing solution. Subsequently, substrate solution (3,3',5,5'-tetramethylbenzidine liquid substrate system for ELISA, Sigma) was 
added and plates were incubated for $20 \mathrm{~min}$ at room temperature. The reaction was stopped with $0.5 \mathrm{M} \mathrm{H}_{2} \mathrm{SO}_{4}$ and plates were read at a wavelength of $450 \mathrm{~nm}$ using an automated plate reader (Multiskan EX, Thermo). The detection limit was about $0.5 \mathrm{ng}$ and was calculated from the mean response of the zero calibrator minus 3x S.D. Intra- and inter-assay variations equaled 5.1 and $9.8 \%$ at the level of $20.2 \mathrm{ng}$ of protein per well.

Sandwich ELISA for estimation of CSF levels of cypD

The levels were estimated using a commercial ELISA kit (MyBiosource). Both intra- and inter-assay variations were less than $15 \%$.

Semiquantitative sandwich ELISA for estimation of CSF levels of $17 \beta$-HSD10 - parkin or $17 \beta-H S D 10$ - cypD complexes

As a capture antibody, we used polyclonal rabbit anti-17 $\beta$-HSD10 (anti-ERAB, Flarebio) that was raised against

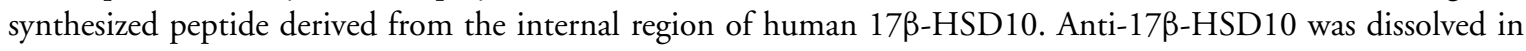
carbonate buffer and the resulting coating solution was applied to 96-well polystyrene plates (Nunc Immuno Plate Maxisorp, $\sim 240 \mathrm{ng}$ of antibody per well). Plates were incubated for $5 \mathrm{~h}$ at room temperature and subsequently washed three-times with phosphate buffer. Nonspecific binding was suppressed by incubation with blocking solution for $2 \mathrm{~h}$ at room temperature followed by overnight incubation at $10^{\circ} \mathrm{C}$. Prior to the assay, the plates were washed three-times with washing solution and incubated in duplicate with $100 \mu \mathrm{l}$ of redistilled water or CSF samples for $5 \mathrm{~h}$ at room temperature (as internal standards, we used a mixture of CSF samples from two SCs [one men and one women]). After incubation, plates were washed three-times with washing solution and primary antibodies (either that against a full-length human PARK2 protein [polyclonal mouse anti-PARK2, Abnova, dilution 1:2000] or that against a full-length human cypD [monoclonal mouse anti-PPID, Abnova, dilution 1:4000]) were applied for $2 \mathrm{~h}$ at room temperature followed by overnight incubation at $10^{\circ} \mathrm{C}$. Plates were then washed four-times with washing solution, and secondary antibody (polyclonal rabbit anti-mouse immunoglobulin/horseradish peroxidase, DakoCytomation, dilution 1:6000) was added. Following procedures were similar to the $17 \beta$-HSD10 experiment. After subtraction of the blank, the values were compared with those from the internal standard and expressed as a percentage. During the development of the methods, parallel experiments with 50 and $80 \mu \mathrm{l}$ of CSF were performed to support the specificity of measurements.

Sandwich ELISA for estimation of CSF levels of AB 1-42

The levels were estimated using a commercial ELISA kit (Invitrogen). Both intra- and inter-assay variations were less than $10 \%$.

Routine analysis of CSF

The number of leukocytes was measured by current microscopy using a Fuchs-Rosenthal's chamber $(3.2 \mu \mathrm{l})$. The total protein concentrations were estimated colorimetrically using the pyrogallol red-molybdate method using a Shimadzu UV-2101PC spectrophotometer. Glucose was estimated by the glucose oxidase method.

\section{Statistical analysis}

Statistical analysis was performed using BMDP statistical software. Analysis of variance (ANOVA) was applied for global and Bonferroni-adjusted t-test (program 7D) or paired t-test (program 3D) for pairwise comparisons. In addition, multiple linear regression (program 1R) and correlation analysis (program 6D) were used. The equality of correlation coefficients in two groups was examined using the test based on Fisher's Z-transformation (Z-test) [29]. Cutoff point and sensitivity/specificity using ROC curve analysis were calculated in MS Excel in accordance with MedCals statistical software manual [30]. Data in the tables are presented as mean \pm SD.

\section{Results}

Table 1 shows the basic characteristics of the 120 study participants (in total, 41 males and 79 females divided into four groups -that is, into 42x SCs, 16x INDCs, 26x MS1 and 36x MS2). Significant differences in their age were not found. Table 2 demonstrates characteristics of 36 patients from MS2 group in more details (patients were divided into two subgroups, i.e., into $16 \mathrm{x}$ relapsing-remitting form in remission phase and $20 \mathrm{x}$ relapsing-remitting form in attack phase). Statistical analysis did not reveal significant differences between MS2 subgroups in the 
Table 3. CSF levels of $17 \boldsymbol{\beta}$-hydroxysteroid dehydrogenase type 10 and amyloid $\boldsymbol{\beta} 1-42$.

\begin{tabular}{|c|c|c|c|}
\hline Groups & $\mathbf{n}$ & $17 \beta$-HSD10 (nM) & $A \beta 1-42(\mathrm{pg} / \mathrm{ml})$ \\
\hline SCs & 42 & $103.2 \pm 16.9$ & $380.5 \pm 131.9$ \\
\hline INDCs & 16 & $106.5 \pm 12.4$ & $413.2 \pm 176.0$ \\
\hline MS1 & 26 & $103.8 \pm 20.4$ & $387.0 \pm 138.0$ \\
\hline MS2 & 36 & $119.4 \pm 21.1^{*, \dagger}$ & $375.0 \pm 137.3$ \\
\hline ANOVA & & $F(3,116)=5.86, p<0.001$ & $F(3,116)=0.29, p=0.834$ \\
\hline
\end{tabular}

Statistical significance (Bonferroni-adjusted t-test) was calculated with respect to SCs (adjusted $p<0.010$ ) or to the MS1 group (adjusted $p<0.010$ ).

Data are presented as mean \pm standard deviation.

* Statistical significance calculated with respect to SCs.

† Statistical significance calculated with respect to the MS1 group.

ANOVA: Analysis of variance; INDC: Inflammatory neurological disease control; MS: Multiple sclerosis; MS1: Patients with MS after the first attack; MS2: Patients with MS after at least two attacks; SC: Symptomatic control.

Figure 1. CSF levels of $17 \beta$-hydroxysteroid dehydrogenase type 10 . Figure shows a part of the results of Table 3 presented as individual value plots. Levels of $17 \beta$-HSD $10(\mathrm{nM})$ were estimated in cerebrospinal fluid samples of SCs $(n=42)$ and MS2 $(n=36)$. Statistical significance (Bonferroni-adjusted t-test) was calculated with respect to SCs group. Data are presented as mean \pm standard deviation.

$* *$ Adjusted $\mathrm{p}<0.010$.

17 $\beta$-HSD10: $17 \beta$-Hydroxysteroid dehydrogenase type 10; MS2: Patients with multiple sclerosis after at least two attacks; SC: Symptomatic control.

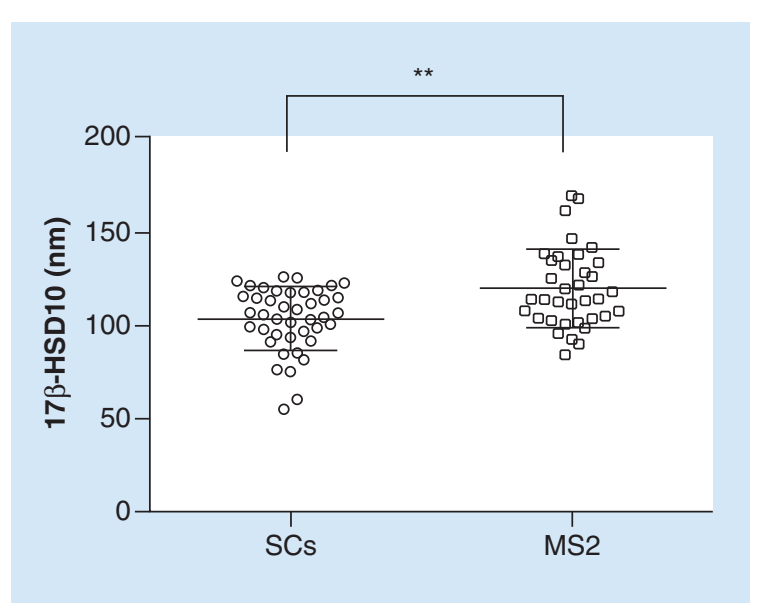

number of attacks at the time of lumbar puncture or in expanded disability status score (EDSS) at the time of lumbar puncture.

Table 3 demonstrates CSF levels of $17 \beta$-HSD10 estimated by competitive ELISA and those of A $\beta$ 1-42 estimated by sandwich ELISA. Significant differences in A $\beta$ 1-42 were not observed among particular groups (ANOVA: $\mathrm{p}=0.834$ ). On the other hand, ANOVA and Bonferroni t-test supported a significant change in $17 \beta-\mathrm{HSD} 10$ in the MS2 group (ANOVA: $p<0.001$, an increase to $115.7 \%$ compared with SCs and to $115.0 \%$ compared with the MS1 group). 17 $\beta$-HSD10 levels estimated in symptomatic controls (SC) and MS2 groups are presented also as individual value plots in Figure 1. Using ROC curve analysis, positive 17 $\beta$-HSD10 levels were defined as values above the cutoff ( $\geq 110.0 \mathrm{nM}$ ). The sensitivity of $17 \beta$-HSD10 to MS in later stages of disease was $63.9 \%$. A comparison with SCs showed the specificity of $62.5 \%$ that with INDCs the specificity of $59.5 \%$.

Significant differences in 17 $\beta$-HSD10 levels between MS2 subgroups were not found (relapsing-remitting form in remission phase: $120.0 \pm 20.2 \mathrm{nM}$, relapsing-remitting form in attack phase: $118.8 \pm 22.2 \mathrm{nM}$, ANOVA: $\mathrm{F}[1,34]=0.03, \mathrm{p}=0.866$ ). Similarly, statistical analysis did not reveal significant differences in A $\beta$ 1-42 levels between MS2 subgroups (relapsing-remitting form in remission phase: $391.0 \pm 132.4 \mathrm{pg} / \mathrm{ml}$, relapsing-remitting form in attack phase: $357.2 \pm 144.5 \mathrm{pg} / \mathrm{ml}$, ANOVA: $\mathrm{F}[1,34]=0.52, \mathrm{p}=0.474)$. Moreover, correlation analysis performed on data from all MS2 patients or separately from MS2 patients in remission phase and MS2 patients in attack phase did not support linear links among levels of $17 \beta-$ HSD 10, levels of A $\beta 1-42$, the number of attacks at the time of lumbar puncture and EDSS at the time of lumbar puncture. Moreover, significant differences between both MS2 subgroups were not found in routine parameters of CSF analyses (the number of leukocytes: $19 \pm 20$ in remission phase, $23 \pm 26$ in acute phase, $\mathrm{p}=0.612$; total proteins $[\mathrm{g} / \mathrm{l}]: 0.30 \pm 0.10$ in remission phase, $0.36 \pm 0.14$ in attack phase, $\mathrm{p}=0.143$; glucose $[\mathrm{mmol} / \mathrm{l}]: 3.14 \pm 0.30$ in remission phase, $3.20 \pm 0.38$ in acute phase, $\mathrm{p}=0.646$ ).

The results of routine analysis of CSF (data not shown) indicated significant differences among particular groups in the number of leukocytes (ANOVA: $\mathrm{p}=0.002$, an increase to $2071.4 \%$ in INDCs compared with SCs) or 
Table 4. CSF levels of 17 $\beta$-hydroxysteroid dehydrogenase type 10-parkin complexes, cyclophilin D and

$17 \beta$-hydroxysteroid dehydrogenase type 10-cyclophilin D complexes.

\begin{tabular}{|c|c|c|c|c|c|}
\hline Groups & $n(M / F)$ & Age (years) & $17 \beta$-HSD10-parkin (\%) & cypD (pg/ml) & $17 \beta$-HSD10-сурD (\%) \\
\hline SCs & $16(5 / 11)$ & $40.8 \pm 7.6$ & $100.0 \pm 12.5$ & $46.9 \pm 15.3$ & $100.0 \pm 34.7$ \\
\hline MS2 & $16(5 / 11)$ & $40.9 \pm 8.8$ & $116.3 \pm 22.5^{*}$ & $41.8 \pm 15.2$ & $89.0 \pm 39.3$ \\
\hline Paired t-test & & $p=0.886$ & $p=0.009$ & $p=0.462$ & $p=0.350$ \\
\hline
\end{tabular}

Age- and sex-matched pairs with CSF samples of sufficiently high volumes were created from totally 16 MS patients after at least two attacks (all MS2 patients with relapsing-remitting form in attack phase) and $16 \mathrm{SCs}$. Data were statistically evaluated by paired t-test.

Data are presented as mean \pm standard deviation.

${ }^{*} P$ value $<0.010$

cypD: Cyclophilin D; F: Female; M: Male; MS: Multiple sclerosis; MS2: Patients with MS after at least two attacks; SC: Symptomatic control.

in total proteins (ANOVA: $\mathrm{p}<0.001$, an increase to $206.7 \%$ in INDCs compared with SCs) but not in the concentration of glucose (ANOVA: $\mathrm{p}=0.357$ ). The results of multiple linear regressions performed separately on the data of SCs, INDCs, MS1 and MS2 groups with one dependent variable (levels of 17 $\beta$-HSD10) and five independent variables (age, A $\beta$ 1-42, leukocytes, total proteins and glucose) revealed a significant linear association only between $17 \beta$-HSD10 and leukocytes in the MS2 group $\left(\mathrm{R}^{2}=0.418\right.$, ANOVA: $\mathrm{F}[5,28]=4.022, \mathrm{p}=0.007$; regression coefficient $=+0.582, \mathrm{p}<0.001)$. No significant result was found in $\mathrm{SCs}\left(\mathrm{R}^{2}=0.055\right.$, ANOVA: $\mathrm{F}[5,36]=0.417, \mathrm{p}=0.834)$, INDCs $\left(\mathrm{R}^{2}=0.190\right.$, ANOVA: $\left.\mathrm{F}[5,10]=0.468, \mathrm{p}=0.792\right)$ or in the MS1 group $\left(\mathrm{R}^{2}=0.134\right.$, ANOVA: $\left.\mathrm{F}[5,20]=0.617, \mathrm{p}=0.689\right)$. The results of multiple linear regressions also supported significant differences between particular groups (ANOVA of regression coefficients over groups: $F[18,94]=2.447$, $\mathrm{p}=0.003)$. Correlation analysis was performed separately on the data of SCs, INDCs, MS1 and MS2 groups and the results can be summarized as follows:

- In SCs, significant positive correlation was found between leukocyte and glucose (correlation coefficient $=+0.321, \mathrm{p}=0.038)$

- In the MS2 group, Z-tests revealed statistically significant shifts from mild positive correlations between $17 \beta$ HSD10 and leukocytes in the SCs/INDCs/MS1 groups to marked negative correlation in MS2 group ( $p=0.020$ compared with SCs, $\mathrm{p}=0.042$ compared with INDCs and $\mathrm{p}=0.012$ compared with MS1 group).

CSF samples of sufficiently high volumes from a total of 32 individuals (16 patients from MS2 group with relapsing-remitting form in attack phase and 16 age- and sex-matched SCs) were used to estimate levels of $17 \beta$ HSD10-parkin complexes, cypD and 17 -HSD10-cypD complexes by sandwich ELISA (Table 4). The levels of $17 \beta$-HSD10-parkin complexes were significantly increased in MS2 groups compared with SCs (the enhancement to $116.3 \%$, paired t-test: $\mathrm{p}=0.009)$. On the other hand, the levels of cypD or $17 \beta-\mathrm{HSD} 10-$ cypD complexes did not significantly differ from each other (paired t-tests: $\mathrm{p}=0.462$ and 0.350 , respectively).

\section{Discussion \\ CSF levels of $17 \beta-$ HSD 10 \& A $\beta$ 1-42 in INDCs}

The results of the study show statistically significant increases in the number of leukocytes and in the total proteins in CSF, both indicating inflammation in the group of INDCs. Although the CSF levels of glucose were not significantly altered, correlation analyses revealed a statistically significant shift from the marked positive correlation between leukocytes and glucose in SCs to the marked negative correlation in INDCs (Results), which could indicate a mild reduction in glucose levels in INDCs probably via activated leukocyte-mediated enhanced glucose consumption during inflammation [31]. In contrast to leukocytes, we have found no significant changes in CSF levels of $17 \beta$-HSD10 in INDCs (Table 3), in accordance with our previous study [18]. The result suggests that $17 \beta$-HSD10 overexpression in the brain observed in both inflammatory AD and MS is not probably based on mitochondria of activated blood leukocytes infiltrating CNS (in the opposite case, the change in $17 \beta$-HSD10 should be high, especially in INDCs). Incidentally, although 17 $\beta$-HSD10 is constitutively expressed in liver, brain and gonadal cells, its localization in mitochondria of leukocytes has not yet been reported [32].

With respect to A $\beta$ 1-42, however, we did not observe a significant change in young INDCs (Table 3), in contrast to our previous study reporting a significant drop to 69.6\% in INDCs compared with SCs [19]. This discrepancy can be explained by marked differences in the age of the study subjects ( $36.9 \pm 1.7$ years in this study, $68.1 \pm 1.9$ 
years in our previous study) since aggregation of $A \beta 1-42$ in vivo is known to be significantly age-dependent, even in healthy people [33].

\section{CSF levels of $17 \beta-H S D 10$ in MS}

The results of the present study support our previous work reporting enhanced CSF levels of 17ß-HSD10 in MS [19], however, only in the patients who had experienced at least two attacks (Table 3). On the other hand, we did not find differences in 17 $\beta$-HSD10 levels between relapsing-remitting form in remission or attack phases (Results), which suggests more permanent changes independently on acute stages of the disease. Because CSF is in direct contact with the brain, increased CSF levels of $17 \beta$-HSD10 should reflect its overexpression in the brain [19-21]. Although detailed mechanisms leading to $17 \beta-H S D 10$ overexpression in MS are not known yet, the results of both multiple linear regression and correlation analysis (Results) show a significant link between 17ß-HSD10 and leukocytes and so indicate a possible role of the immune system.

It is well known that leukocytes of both the adaptive immune system (e.g., $\mathrm{CD}^{+} \mathrm{T}$ and $\mathrm{CD}^{+} \mathrm{T}$ lymphocytes or B lymphocytes) and the innate immune system (e.g., natural killer cells or mast cells) are involved in the pathogenesis of MS, some of them especially in its relapsing-remitting stage [1,2,4-8]. Experiments on blood samples have revealed that some leukocytes are activated or elevated, while other leukocytes can be dysfunctional or deficient. Moreover, activated peripheral blood lymphocytes (probably especially T and B cells) of untreated relapsing-remitting MS patients show different ultrastructural characteristics, including an increased number/size of mitochondria compared with controls [34]. However, 17/-HSD10 overexpression in MS is probably not based on mitochondria of the activated blood leukocytes crossing the blood-brain barrier and infiltrating the CNS (see no changes in INDCs), but it is more likely due to mitochondria of damaged neuronal axons. In accordance with many studies reporting the protective role of $17 \beta-\mathrm{HSD} 10$ in oxidative/metabolic stress and PD [9-11], as well as the increased mitochondrial density in neuronal axons in all MS lesions [2,4], we suppose that 17/-HSD10 overexpression in later stages of MS could be interpreted via upregulation of the protein in demyelinated neuronal axons as a protective compensatory mechanism against attacts of the body's own immune system. On the other hand, 17 $\beta$-HSD10 is present in the brain tissue not only in neurons but also in glia or in smooth muscle cells [35] and thus a possible involvement of non-neuronal cells in $17 \beta-\mathrm{HSD} 10$ overexpression should not be omitted althogether.

However, it is not clear if the upregulation of $17 \beta-H S D 10$ leads to the increased concentrations of $17 \beta-H S D 10$ in the mitochondrial matrix. With respect to the high sensitivity of the Pink1 (parkin) the translocase of the outer mitochondrial membrane/the translocase of the inner mitochondrial membrane pathway to oxidative stress $[12,13]$ and to the links among immune system-inflammation-oxidative stress in MS [4], the upregulation of the protein in demyelinated neuronal axons does not have to be followed by its increased transport into mitochondria. In order to study conditions in mitochondria in later stages of MS in more details, we evaluated abilities either of parkin protein to regulate mitochondrial levels of $17 \beta-$ HSD10 via estimations of CSF levels of $17 \beta-H S D 10$ parkin complexes either of $17 \beta$-HSD10 to regulate cypD-mediated opening of MPTP in the mitochondrial matrix via estimations of CSF levels of cypD and 17 $\beta$-HSD10 - cypD complexes. Measurements based on sandwich ELISA method were performed in accordance with our previous studies evaluating interactions of two endogenous substances $[19,20]$. Since our results revealed increased concentrations of $17 \beta-H S D 10$ in CSF (Table $3 \&$ Figure 1 )

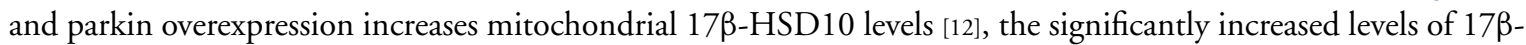
HSD10-parkin complexes but no significant changes in cypD and 17 $\beta$-HSD10-cypD complexes (Table 4) could be interpreted via enhanced concentrations of functional $17 \beta-\mathrm{HSD} 10$ in the mitochondrial matrix in later stages

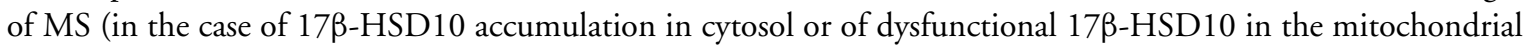
matrix, concentrations of 17 $\beta$-HSD10-cypD complexes should be decreased). On the other hand, it is important to note that CSF analyses do not distinguish between originally cytosolic or mitochondrial 17 $\beta$-HSD10 and that,

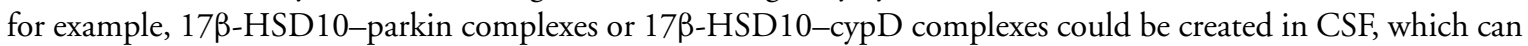
limitate data obtained in similar analyses.

With respect to secondary mitochondrial dysfunction observed in people with MS [4,5] and to a negative role of high levels of $17 \beta-H S D 10$ in the mitochondrial matrix [18], the pathway leading to highly overexpressed $17 \beta-$ HSD10 in the mitochondrial matrix could be one of mechanisms involved in mitochondrial dysfunction in later stages of MS. Nevertheless, the increase in $17 \beta$-HSD10 levels to $115.7 \%$ (Table 3) is not very robust and so its impact on mitochondrial dysfunction should not be crucial (see also no links between 17 $\beta$-HSD10 levels and EDSS in patients in later stages of MS, for example). 
The questionable role of $A \beta$ in the pathogenesis of MS

All our results obtained in CSF samples indicate that $A \beta$ should not play a key role in the pathogenesis of MS. For example, there were no changes in A $\beta 1-42$, no links between $17 \beta-H S D 10$ and $A \beta 1-42$ via multiple linear regression or correlation analyses. Plus, there were no links between $A \beta 1-42$ and the number of attacks at the time of lumbar puncture or EDSS at the time of lumbal puncture via correlation analyses (all in this study), also no changes in Thioflavin T-based fluorescence indicating no alterations in peptide/protein aggregates [36]. Finally there were no changes in oligomeric $A \beta$ and no alterations in $17 \beta-H S D 10-A \beta$ complexes [19]). Nevertheless, MS and $\mathrm{AD}$ are both progressive neurodegenerative and neuroinflammatory disorders leading to memory impairment. Therefore, a potential role of $A \beta 1-42$, in other words, of the classical CSF biomarker for $A D$, is often discussed in relation to MS [22,23,37-40]. Experimental results are controversial since no significant changes in this study [37,39] as well as alterations similar to those seen in $\mathrm{AD}[22,23,38]$ were found. Discrepancies could partly be based on the different stages of MS (see significant changes in MS during relapse [38]), on differences in age of patients (see CSF levels of $A \beta 1-42$ in young INDCs in this study and in old INDCs [20]) and finally on the type of treatment (see reductions or increases in A $\beta$ before and after treatment of MS [22]). Despite of our results, we suppose that the pronounced role of the immune system in the development of MS (e.g., $[1,2])$ and the function of $A \beta$ in the innate immune system (e.g., [41]) could enhance a probability of A $\beta$ involvement in MS.

\section{CSF levels of $17 \beta-$ HSD10 as a biomarker for MS}

Some studies have reported the similar alterations of classical biomarkers for AD in MS [22,23,38]; the relatively high sensitivity of $17 \beta$-HSD10 levels in CSF for early stages of AD [20] and finally, increased CSF levels of $17 \beta$-HSD10 in $\mathrm{AD}$ and MS [19]. Therefore, we decided to evaluate CSF levels of $17 \beta-H S D 10$ as a prospective biomarker for MS. However, results of the study suggest that it was not a good choice. First, the levels are not the valuable biomarker for the early diagnosis of MS (see no changes in early stages of MS, in contrast to AD [19-21]). Second, the levels are not the valuable biomarker for the progression of the neurodegenerative component of MS (see no differences in the levels between relapsing-remitting form in remission or attack phases, no correlation between the levels and the number of attacks or between those and EDSS). And finally, the sensitivity of $64 \%$ found in later stages of MS and the specificity of $63 \%$ in a comparison with SCs or of $60 \%$ in a comparison with inflammatory neurological disease controls appear to be lower when compared with $\mathrm{AD}$.

\section{Conclusion}

We have found the significant increases in levels of $17 \beta$-HSD10 or of 17 -HSD10-parkin complexes and the links between $17 \beta-H S D 10$ and leukocytes in CSF samples of patients with relapsing-remitting MS. No alterations were observed in levels of $\mathrm{A} \beta 1-42$, cypD or 17 $\beta$-HSD10-cypD complexes. Moreover, no links between $17 \beta-\mathrm{HSD} 10$ levels and $A \beta 1-42 /$ the number of attacks/EDSS were revealed. Data could be interpreted via upregulation of the protein in demyelinated neuronal axons as a protective compensatory mechanism against attacks of the body's own immune system. It seems that the upregulation of $17 \beta$-HSD10 could lead to increased concentrations of the functional protein in the mitochondrial matrix but that the impact on mitochondrial dysfunction should not be crucial. CSF levels of $17 \beta$-HSD10 do not appear to be the valuable biomarker for early diagnosis or for progression of MS.

\section{Summary points}

- Multiple sclerosis (MS) is characterized among others by secondary mitochondrial dysfunction.

- Overexpressed 17 $\beta$-hydroxysteroid dehydrogenase type 10 (17 $\beta$-HSD10) may lead to mitochondrial dysfunction.

- Increased levels of 17/-HSD10 were observed in CSF of people with MS, however, not in early stages.

- CSF levels of the protein in people with MS do not reflect differences between remission and attack phases.

- CSF levels of the protein in people with MS do not correlate with expanded disability status score.

- CSF levels of the protein do not correlate with levels of amyloid $\beta$ 1-42.

- Regulation of cyclophilin D by $17 \beta-H S D 10$ is not probably altered in MS.

- $17 \beta-H S D 10$ is not the valuable biomarker for the diagnosis of MS. 


\section{Open access}

This work is licensed under the Attribution-NonCommercial-NoDerivatives 4.0 Unported License. To view a copy of this license, visit http://creativecommons.org/licenses/by-nc-nd/4.0/

\section{Financial \& competing interests disclosure}

This study was supported by Ministry of Health of the Czech Republic (16-27611A project), by the Czech Science Foundation (P304-12G069 project), by the MEYS under the NPU I program (project No. L01611) and by Charles University in Prague (Progres Q35 project). The authors declare that the research was conducted in the absence of any commercial or financial relationships that could be constructed as a potential conflict of interest.

No writing assistance was utilized in the production of this manuscript.

\section{Ethical conduct of research}

The authors state that they have obtained appropriate institutional review board approval or have followed the principles outlined in the Declaration of Helsinki for all human or animal experimental investigations. In addition, for investigations involving human subjects, informed consent has been obtained from the participants involved. All subjects gave written informed consent regarding study participation, in accordance with the Declaration of Helsinky. The Ethics Committee of the Third Faculty of Medicine, Charles University in Prague approved the study.

\section{References}

1. Frischer JM, Bramow S, Dal-Bianco A et al. The relation between inflammation and neurodegeneration in multiple sclerosis brains. Brain 132(5), 1175-1189 (2009).

2. Yadav SK, Mindur JE, Ito K, Dhib-Jalbut S. Advances in the immunopathogenesis of multiple sclerosis. Curr. Opin. Neurol. 28(3), 206-219 (2015).

3. Van Horssen J, van Schaik P, Witte M. Inflammation and mitochondrial dysfunction: a vicious circle in neurodegenerative disorders? Neurosci. Lett. pii: S0304-3940(17) 30542-30546 (2017).

4. Carvalho KS. Mitochondrial dysfunction in demyelinating diseases. Semin. Pediatr. Neurol. 20(3), 194-201 (2013).

5. Morató L, Bertini E, Verrigni D et al. Mitochondrial dysfunction in central nervous system white matter disorders. Glia 62(11), 1878-1894 (2014).

6. Witte ME, Mahad DJ, Lassmann H, van Horssen J. Mitochondrial dysfunction contributes to neurodegeneration in multiple sclerosis. Trends Mol. Med. 20(3), 179-187 (2014).

7. Morris G, Berk M. The many roads to mitochondrial dysfunction in neuroimmune and neuropsychiatric disorders. BMC Med. 13, 68 (2015).

8. Errea O, Moreno B, Gonzalez-Franquesa A et al. The disruption of mitochondrial axonal transport is an early event in neroinflammation. J. Neuroinflamm. 12, 152 (2015).

9. Yang SY, He XY, Isaacs C et al. Roles of $17 \beta$-hydroxysteroid dehydrogenase type 10 in neurodegenerative disorders. J. Steroid. Biochem. Mol. Biol. 143, 460-472 (2014).

10. Tieu K, Perier C, Vila M et al. L-3-hydroxyacyl-CoA dehydrogenase II protects in a model of Parkinson's disease. Ann. Neurol. 56(1), 51-60 (2004).

11. Yan SD, Stern DM. Mitochondrial dysfunction and Alzheimer's disease: role of amyloid- $\beta$ peptide alcohol dehydrogenase (ABAD). Int. J. Exp. Pathol. 86(3), 161-171 (2005).

12. Bertolin G, Jacoupy M, Traver $S$ et al. parkin maintains mitochondrial levels of the protective Parkinson's disease-related enzyme 17- $\beta$ hydroxysteroid dehydrogenase type 10. Cell Death Differ. 22(10), 1563-1576 (2015).

13. Joselin AP, Hewitt SJ, Callaghan SM et al. ROS-dependent regulation of parkin and DJ-1 localization during oxidative stress in neurons. Hum. Mol. Genet. 21(22), 4888-4903 (2012).

14. Muirhead KEA, Borger E, Aitken L et al. The consequences of mitochondrial amyloid $\beta$-peptide in Alzheimer's disease. Biochem. J. 426(3), 255-270 (2010).

15. Carlson EA, Marquez RT, Du F et al. Overexpression of $17 \beta$-hydroxysteroid dehydrogenase type 10 increases pheochromocytoma cell growth and resistance to cell death. BMC Cancer 15, 166 (2015).

16. Hovorkova P, Kristofikova Z, Horinek A et al. Lateralization of $17 \beta$-hydroxysteroid dehydrogenase type 10 in hippocampi of demented and psychotic people. Dement. Geriatr. Cogn. Disord. 26(3), 193-198 (2008).

17. Rauschenberger K, Schöler K, Sass JO et al. A non-enzymatic function of $17 \beta$-hydroxysteroid dehydrogenase type 10 is required for mitochondrial integrity and cell survival. EMBO Mol. Med. 2(2), 51-62 (2010). 
18. Shafqat N, Marschall HU, Filling C et al. Expanded substrate screenings of human and Drosophila type 10 17ß-hydroxysteroid dehydrogenases (HSDs) reveal multiple specific in bile acid and steroid hormone metabolism: characterization of multifunctional $3 \alpha / 7 \alpha / 7 \beta / 17 \beta / 20 \beta / 21-H S D$. Biochem. J. 376(1), 49-60 (2003).

19. Kristofikova Z, Bockova M, Hegnerova $\mathrm{K}$ et al. Enhanced levels of mitochondrial enzyme $17 \beta$-hydroxysteroid dehydrogenase type 10 in patients with Alzheimer disease and multiple sclerosis. Mol. Biosyst. 5(10), 1174-1179 (2009).

20. Kristofikova Z, Ripova D, Bartos A et al. Neuroinflammation and complexes of $17 \beta$-hydroxysteroid dehydrogenase type 10 - amyloid $\beta$ in Alzheimer's disease. Curr. Alzheimer. Res. 10(2), 165-173 (2013).

21. Kristofikova Z, Ricny J, Vyhnalek M et al. Levels of 17ß-hydroxysteroid dehydrogenase type 10 in cerebrospinal fluid of people with mild cognitive impairment and various types of dementias. J. Alzheimers Dis. 48(1), 105-114 (2015).

22. Augutis K, Axelsson M, Portelius E et al. Cerebrospinal fluid biomarkers of $\beta$-amyloid metabolism in multiple sclerosis. Mult. Scler 19(5), 543-552 (2013).

23. Pietroboni AM, Schiano di Cola F, Scaroni M et al. CSF $\beta$-amyloid as a putative biomarker of disease progression in multiple sclerosis. Mult. Scler. 23(8), 1085-1091 (2017).

24. Hares K, Wilkins A. Axonal transport proteins as biomarkers of neurodegeneration? Biomark. Med. 11(8), 589-591 (2017).

25. Polman CH, Reingold SC, Banwell B et al. Diagnostic criteria for multiple sclerosis: 2010 revision to the McDonald criteria. Ann. Neurol. 69(2), 292-302 (2011).

26. Teunissen C, Menge T, Altintas A et al. Consensus definitions and application guidelines for control groups in cerebrospinal fluid biomarker studies in multiple sclerosis. Mult. Scler. J. 19(13), 1802-1809 (2013).

27. Deisenhammer F, Bartos A, Egg R et al. Guidelines on routine cerebrospinal fluid analysis. Report from an EFNS task force. Eur. J. Neurol. 13(9), 913-922 (2006).

28. Teunissen CE, Petzold A, Benett JL et al. A consensus protocol for the standardization of cerebrospinal fluid collection and biobanking Neurology 73(22), 1914-1922 (2009).

29. Rao CR. Linear Statistical Inference and Its Applications (2nd Edition). John Wiley \& Sons, NY, USA (2002).

30. Schoonjans F. MedCalc Manual: Easy-to-Use Statistical Software. MedCalc Software, Ostend, Belgium (2017).

31. Xia CQ, Chernatynskaya AV, Looney B et al. Anti-CD3 antibody treatment induces hypoglycemia and super tolerance to glucose challenge in mice through enhancing glucose consumption by activated lymphocytes. J. Immunol. Res. 2014, 326708 (2014).

32. Yang SY, He XY, Schultz H. Multiple functions of type 10 17ß-hydroxysteroid dehydrogenase. Trends Endocrin. Met. 16(4), 167-175 (2005).

33. Fukumoto $\mathrm{H}$, Asami-Odaka A, Suzuki $\mathrm{N}$ et al. Amyloid beta protein deposition in normal aging has the same characteristics as that in Alzheimer's disease. Predominance of A beta 42(43) and association of A beta 40 with cored plaques. Am. J. Pathol. 148(1), 259-265 (1996).

34. Djaldetti R, Achiron A, Ziv I, Djaldetti M. Lymphocyte ultrastructure in patients with multiple sclerosis. Biomed. Pharmacother. 49(6), 300-303 (1995).

35. Frackowiak J, Mazur-Kolecka B, Kaczmarski W, Dickson D. Deposition of Alzheimer's vascular amyloid- $\beta$ is associated with decreased expression of brain L-3-hydroxyacyl-coenzyme A dehydrogenase (ERAB). Brain Res. 907(1-2), 44-52 (2001).

36. Kristofikova Z, Gazova Z, Siposova $\mathrm{K}$ et al. Effects of ferrofluid and phytoalexin spirobrassinin on Thioflavin-T-based fluorescence in cerebrospinal fluid of the elderly and multiple sclerosis patients. Neurochem. Res. 39(8), 1502-1510 (2014).

37. Mai W, Hu X, Lu Z et al. Cerebrospinal fluid levels of soluble amyloid precursor protein and $\beta$-amyloid 42 in patients with multiple sclerosis, neuromyelitis optica and clinically isolated syndrome. J. Int. Med. Res. 39(6), 2402-2413 (2011).

38. Mitosek-Szewczyk K, Gordon-Krajcer W, Flis D, Stelmasiak Z. Some markers of neuronal damage in cerebrospinal fluid of multiple sclerosis patients in relapse. Folia Neuropathol. 49(3), 191-196 (2011).

39. Szalardy L, Zadori D, Simu M et al. Evaluating biomarkers of neuronal degeneration and neuroinflammation in CSF of patients with multiple sclerosis - osteopontin as a potential marker of clinical severity. J. Neurol. Sci. 331(1-2), 38-42 (2013).

40. Gentile A, Mori F, Bernardini S, Centonze D. Role of amyloid- $\beta$ CSF levels in cognitive deficit in MS. Clin. Chim. Acta 449(2565929), $123-130(2015)$.

41. Soscia SJ, Kirby JE, Washicosky KJ et al. The Alzheimer's disease-associated amyloid beta-protein is an antimicrobial peptide. PLoS ONE 5(3), e9505 (2010). 